\title{
LETTERS \\ Determinants and Outcomes of Nephrology Care
}

\author{
Milda R. Saunders, M.D., M.P.H. ${ }^{7}$ and Monica B. Vela, M.D. ${ }^{2}$ \\ ${ }^{\text {} H o s p i t a l ~ M e d i c i n e, ~ U n i v e r s i t y ~ o f ~ C h i c a g o ~ M e d i c i n e, ~ C h i c a g o, ~ I L, ~ U S A ; ~}{ }^{2}$ General Internal Medicine, University of Chicago Medicine, Chicago, IL, USA.
}

J Gen Intern Med 31(6):596

DOI: $10.1007 / \mathrm{s} 11606-016-3634-1$

(c) Society of General Internal Medicine 2016

To the Editors:

We read with interest the findings of the important study "Influence of Nephrologist Care on Management and Outcomes in Adults with Chronic Kidney Disease" by Ricardo et al. ${ }^{1}$ The authors found that over two-thirds of almost 4000 participants in the Chronic Renal Insufficiency Cohort (CRIC) with chronic kidney disease (CKD) had seen a nephrologist prior to study enrollment. Prior work has shown the rates of nephrology care for patients with CKD Stages 3-4 ranges from 11 to $39 \% .^{2-4}$ Receipt of nephrology care was often driven by clinical indications (lower eGFR, proteinuria) and clinical judgment (older patients less likely). Clinical risk factors associated with CKD progression - diabetes and obesity - were actually associated with a lower likelihood of nephrology care. Men, those with college education and the insured were more likely to receive nephrology care, even after controlling for clinical factors. Additionally, although prior nephrology contact was not associated with CKD progression, CV events or death, this study did not assess other important outcomes, such as patient knowledge or preparation for dialysis or transplant.

This study highlights several important considerations. One, physician awareness is important. In order to be referred to the CRIC study, the physician had to know the patient had CKD. Consistent with prior work, nephrology referral and receipt of guideline recommended care increased if physicians noted CKD in the problem list, a proxy for awareness of patients' CKD. ${ }^{4}$ Two, networks matter for clinical care. Individuals in CRIC received care at academic medical centers (AMCs) or community practices with ties to AMCs. These connections likely improved health care access and clinical

Published online February 222016 care. Over half of patients with an eGFR $<60$ met guidelinerecommended goals for CKD care, independent of prior contact with nephrology. ${ }^{5}$ Three, this work is hard. A significant proportion of these patients still did not have adequate blood pressure, phosphate or iPTH control. Finally, even in this cohort, disparities in receipt of nephrology care were noted based on age, race, gender and SES. This study did not examine how those patient factors were associated with receiving guideline recommended CKD care.

CKD continues to be a growing issue as the prevalence of diabetes and hypertension increases and the population ages. This work is hard and requires a partnership between patients, generalists and subspecialists to meet guideline-recommended care for all patients with CKD.

\section{REFERENCES}

1. Ricardo AC, Roy JA, Tao K, et al. Influence of nephrologist care on management and outcomes in adults with chronic kidney disease. J Gen Intern Med. 2016;31(1):22-29.

2. Navaneethan S, Kandula $\mathbf{P}$, Jeevanantham V, Nally $\mathbf{J} \mathbf{J r}$, Liebman S. Referral patterns of primary care physicians for chronic kidney disease in general population and geriatric patients. Clin Nephrol. 2010;73(4):260-267.

3. Allen AS, Forman JP, Orav EJ, Bates DW, Denker BM, Sequist TD. Primary care management of chronic kidney disease. J Gen Intern Med. 2011;26(4):386-392.

4. Jolly SE, Navaneethan SD, Schold JD, et al. Chronic kidney disease in an electronic health record problem list: quality of care, ESRD, and mortality. Am J Nephrol. 2014;39(4):288-296.

5. Inker LA, Astor BC, Fox CH, et al. KDOQI US commentary on the 2012 KDIGO clinical practice guideline for the evaluation and management of CKD. Am J Kidney Dis. 63(5):713-735. 